\title{
Improving multidisciplinary meetings in geriatric evaluation and management units
}

\author{
Ken GW Teo ${ }^{1}$, MB BS (Hons), Wei Wang ${ }^{2}$, PhD (Public Health), MSc (Applied \\ Statistics), Anjali Khushu' ${ }^{1}$, MB BS, FRACP \\ 1 Department of Geriatric Medicine, Peninsula Health, Melbourne, Australia \\ 2 Peninsula Clinical School, Monash University, Melbourne, Australia \\ Correspondence to: Ken GW Teo, Department of Geriatric Medicine, Peninsula Health, \\ 24 Separation Street, Mornington, Victoria 3931, Australia.Email: kenteogw@gmail.com
}

\section{To the Editor:}

With an ageing population and an increasing number of frail older adults, ${ }^{1}$ geriatric evaluation and management (GEM) is an increasingly important field. GEM involves a multidisciplinary team to restore functional ability or enhance residual functional capability in older adults with disabling impairments. These impairments are associated with medical conditions related to ageing, including falls, incontinence, reduced mobility, delirium, and dementia. $^{2}$

Multidisciplinary meetings are a core and standard feature in GEM. This formal coordination between various disciplines (geriatricians, physiotherapists, occupational therapists, social workers, speech therapists, dieticians, and nurses) is usually held weekly to comprehensively assess the needs of patients and develop an individualised plan.

We evaluated the quality and effectiveness of multidisciplinary meetings in four units at Peninsula Health, Melbourne, Australia. First, electronic health records of 80 patients discharged from the four units were reviewed retrospectively in February 2018. The interdisciplinary care plan and multidisciplinary discharge summary were evaluated in terms of the quality of goal setting using the SMART (specific, measurable, achievable, relevant and time-framed) criteria. ${ }^{3}$ Second, meeting attendees from each health discipline completed a standardised survey. The survey covered pre-meeting preparation, timeliness, focus, tone, follow-up of issues post meeting, interruptions, leadership, problem solving, respectful challenge, and consensus. Responses were measured on a Likert scale from 'strongly disagree' to 'strongly agree'. There was also an open question: "How can we improve our multidisciplinary meetings?"

A total of 80 patient records and 800 goals were analysed. Most goals were achievable and relevant, but only a small proportion of goals were specific $(28.0 \%)$, measurable $(29.1 \%)$, and time-framed $(22.1 \%)$.

A total of 38 (85\%) staff members responded to the survey. For preparation, $94.4 \%$ of participants agreed or strongly agreed that they knew what was expected of them in advanced and were ready to contribute. The interdisciplinary care plan was used to document the goals and progress of patient. $50.8 \%$ of participants disagreed or strongly disagreed that these were populated prior to the meeting. For timing, 55.3\% of participants agreed or strongly agreed that meetings started and ended on time with segments running close to budgeted time. For focus, $84.2 \%$ of participants agreed or strongly agreed that meeting members stayed focused on the issue at hand. For tracking, $89.2 \%$ of participants agreed or strongly agreed that meetings stayed on track and followed the agenda. For tone, $97.4 \%$ of participants agreed or strongly agreed that the tone of meetings was positive regardless of topic. For follow-up, $86.5 \%$ of participants agreed or strongly agreed that there was a plan to deal with issues that didn't get resolved or were out of the scope of the meeting. For interruptions, $45.9 \%$ of participants disagreed or strongly disagreed that meetings were being disrupted owing to people leaving, phones ringing, pagers beeping, etc. For leadership, $65.8 \%$ 
of participants agreed or strongly agreed that the meetingleader(s) effectively moderated the meetings. However, the responses were diverse in terms of the person or discipline leading the meeting: $16.7 \%$ for nurses, $22.2 \%$ for doctors, $36.1 \%$ for nurses and doctors (co-chair), and $25.0 \%$ for others. For problem solving, $97.4 \%$ of participants agreed or strongly agreed that the group generally solved problem well and produced solutions at meetings. For respectful challenge, $89.2 \%$ of participants agreed or strongly agreed that there was respectful challenge of goals set during the meeting. For consensus, 100\% of participants agreed or strongly agreed the team worked hard to make collaborative decisions.

Answers to the open question of how to improve multidisciplinary meetings included (1) more individualised SMART goals for patients, (2) clearly identify what is required for discharge and set goals to that, (3) start meeting on time, (4) pre-fill interdisciplinary care plan with goals and patient progress, (5) clear leader/chairperson for meeting, and (6) effective communication between team members outside of meeting so that holistic decisions can made in a timely fashion.

Most goals were not specific, measurable, or timeframed, probably because of a lack of training with regards to goal setting. However, most goals were achievable and relevant, indicating that clinicians had a holistic view of the patient circumstances and abilities and appreciated the achievable and relevant components better.

The multidisciplinary meeting was effective in most aspects apart from the interdisciplinary care plan not filled prior to the meeting, meetings not starting on time, interruptions during meeting, and lack of a clear chairperson.

Several strategies may improve multidisciplinary meetings. First, more education is needed regarding the setting of SMART goals. A good-quality goal reduces the duration of meetings and makes meetings more fruitful. More attention should be given to the specific, measurable, and time-framed aspects. Having an information sheet about SMART goals on the meeting table for easy reference or providing clinicians with a template for SMART goal setting could facilitate this process. Second, a clear leader or chairperson of the meeting should be appointed.Third, it is important to pre-fill care plans that documents patient goals and progress to allows the meeting to run more efficiently. Lastly, as most multidisciplinary meetings run weekly, it is important for staff members to communicate effectively outside the meeting to ensure timely decisions are made.

Our study is the first to evaluate multidisciplinary meetings in GEM units. Although it is based on a single site, the knowledge gained and strategies proposed to improve multidisciplinary meetings are applicable to other units.

\section{DECLARATION}

The authors have no conflicts interest to disclose.

\section{REFERENCES}

1. Lutz W, Sanderson W, Scherbov S. The coming acceleration of global population ageing. Nature 2008;451:716-9. Crossref

2. Rubenstein LZ, Stuck AE, Siu AL, Wieland D. Impacts of geriatric evaluation and management programs on defined outcomes: overview of the evidence.J Am Geriatr Soc 1991;39:8S-18S. Crossref

3. Bovend'Eerdt TJ, Botell RE, Wade DT. Writing SMART rehabilitation goals and achieving goal attainment scaling: a practical guide. Clin Rehabil 2009;23:352-61. Crossref 